\title{
Não há nada tão ruim que não possa piorar: uma análise Bayesiana da pobreza
}

\author{
Rodolfo Ferreira Ribeiro da Costa \\ Universidade do Estado do Rio Grande do Norte - Mossoró - RN - Brasil \\ ORCID: 0000-0002-9761-5858
}

\begin{abstract}
Resumo
Este artigo visa compreender as relações existentes entre diferentes formas de pobreza e, assim, verificar se um determinado tipo de carência inibe a capacidade de o indivíduo superar de outras formas de privações. A abordagem utilizada para entender os mecanismos que regem as relações entre distintos níveis de pobreza remete-se a rede bayesiana. Os dados utilizados referem-se às informações sobre renda, saúde, educação e habitação para 5.565 municípios brasileiros entre 1970 e 2010, sendo a fonte de tais informações devida ao Instituto Brasileiro de Geografia e Estatística. Os resultados destacam a existência de relações entre as facetas da pobreza analisadas. Foram observadas influências diretas de todas as privações sobre as condições de saúde, indicando que precariedades em saúde normalmente surgem em ambientes onde outros tipos de carências afloram. $O$ indicador de educação reflete informações sobre a privação monetária, sendo tal relação de caráter positivo. Por fim, carências monetárias são responsáveis por limitar, também, o acesso a uma moradia digna e a uma condição de vida mais salutar.
\end{abstract}

Palavras-chave: Pobreza. Rede Bayesiana. Municípios.

There is nothing so bad that it can not work: a Bayesian analysis of poverty

\section{Abstract}

this article aims to understand the relationships between different forms of poverty and, thus, to verify if a specific type of deprivation inhibits the individual's ability to overcome other forms of deprivation. An approach used to understand the mechanisms that govern how the relations between the levels of poverty refer to the Bayesian network. The data used refer to information on income, health, education and housing for 5,565 Brazilian municipalities between 1970 and 2010, being a source of information provided by the Brazilian Institute of Geography and Statistics. The results highlighted the existence of relations between the analyzed aspects of poverty. Direct influences of all deprivations on health conditions were observed, indicating that precarious health conditions usually arise in environments where other types of diseases arise. The education indicator reflects information on monetary deprivation, and this relationship is positive. Finally, monetary shortages are also responsible for limiting access to decent housing and a healthier living condition.

Keywords: Poverty. Network Bayesian. Municipals. 


\section{Resumen \\ No hay nada tan ruim que no puede piorar: un análisis Bayesiana de la pobreza}

Este artículo pretende comprender las relaciones existentes entre diferentes formas de pobreza y, así, verificar si un determinado tipo de carencia inhibe la capacidad del individuo de superar de otras formas de privaciones. El enfoque utilizado para entender los mecanismos que rigen las relaciones entre distintos niveles de pobreza se remite a la red bayesiana. Los datos utilizados se refieren a las informaciones sobre renta, salud, educación y vivienda para 5.565 municipios brasileños entre 1970 y 2010, siendo la fuente de tales informaciones debida al Instituto Brasileño de Geografía y Estadística. Los resultados destacan la existencia de relaciones entre las facetas de la pobreza analizadas. Se observaron influencias directas de todas las privaciones sobre las condiciones de salud, indicando que precarias en salud normalmente surgen en ambientes donde otros tipos de carencias afloran. Otro hecho importante es que las privaciones educativas no son explicadas por ninguna de las ópticas tratadas y, por lo tanto, retratan un carácter exógeno. El indicador de educación también refleja información sobre la privación monetaria, siendo tal relación de carácter positivo. Por último, las carencias monetarias son responsables de limitar el acceso a una vivienda digna.

Palabras clave: La pobreza. Red Bayesiana. Municipios.

\section{Introdução}

O conceito de pobreza multidimensional ganhou destaque entre pesquisadores e formuladores de políticas principalmente a partir de Sen (1976), que tratou a pobreza em múltiplos sentidos, e da elaboração das Metas do Milênio, que destacavam as privações sofridas pelos indivíduos. A literatura tem tratado a pobreza como sendo o estado de limitação no qual os indivíduos, famílias ou grupos apresentam uma condição monetária abaixo da necessária para garantir um padrão de vida minimamente aceitável e, portanto, as considerações sobre a ótica multidimensional seriam dadas a partir da extrapolação do critério de forma a incluir fatores não monetários, tais como: saúde, educação, habitação etc.

Os estudos direcionados a mensuração da pobreza multidimensional tem crescido consideravelmente. Trabalhos como Bourguignon e Chakravarty (2003) e Tsui (2002), Alkire e Foster (2011), Chakravarty, Deutsch e Silber (2008), Duclos, Sahn e Younger (2006), Maasoumi e Lugo (2008), Kolm (1977), Atkinson e Bourguignon (1982), Maasoumi (1986), Tsui (1995), Greer e Thorbecke (2010), Bibi (2005) e Bossert, Chakravarty e D’Ambrosio (2009) propõem uma série de abordagens para medir ou analisar privações em mais de uma dimensão.

Neste contexto, severas são as críticas em relação ao uso de indicadores monetários individuais para análise da pobreza, já que as pessoas com pobreza monetária não necessariamente sofrem com privações não monetárias. RuggeriLaderchi, Saith e Stewart (2003) observaram que, na Índia, $43 \%$ das crianças e mais da metade dos adultos enquadrados como pobres em educação ou saúde não estavam dentre aqueles com pobreza monetária. Segundo Bourguignon e Chakravarty (2003), a pobreza depende de variáveis monetárias e não monetárias. Uma maior renda pode ser capaz de melhorar a posição de alguns de seus atributos não monetários e, portanto, utilizar a renda como único indicador de pobreza provavelmente seria uma ação inadequada, devendo o mesmo ser 
complementado por outros atributos, tais como habitação, alfabetização, expectativa de vida e bens públicos.

Ainda, mesmo diante de considerações sobre diversas carências, à análise da pobreza normalmente é balizada na construção de indicadores computados através de agregações feitas a partir de poderações, quase sempre ad hoc, das distintas privações análisadas, como é o caso do IDH, IPF, IPH e o IDF, e que, ainda, apresentam questões controvérsas em relação à definição da linha de pobreza. Tais arbitrariedades, como indicam Cheli e Lemmi (1995) e Cerioli e Zani (1990), poderam ser superadas a partir de indicadores obtidos através da fuzzy sets approach. Contudo, esta medida possibilita uma análise relativa das condições, ponderando as privações de forma objetiva, sem refletir o ciclo de relações existentes entre as mesmas.

Portanto, a literatura tem destacado que os resultados observados para indicadores de pobreza multidimensional parecem negligenciar as relações existentes entre as privações. Tal fato restringe a compreensão de como um determinado estado de carência pode contribuir para as condições gerais da vida de um indivíduo. Analisar as relações entre as distintas dimensões da pobreza podem fornecer uma interpretação mais precisa sobre as armadilhas inerentes as carências e, assim, tornar o entendimento das condições de vida mais compreensivo.

Diversos trabalhos confirmam a existência de modalidades de pobreza e, ainda, são capazes de identificar seus determinantes, contudo poucos se detêm a entender como funciona o mecanismo interativo entre as privações sem impor restrições a relação de causalidade ou mesmo incorporar a relação condicional entre suas respectivas distribuições. Neste sentido, o objetivo deste trabalho é identificar as relações existentes entre os diferentes tipos de pobreza e, assim, compreender como um dado tipo de carência pode ser responsável por afetar as demais formas de privações. O estudo das relações entre as privações dar-se-á através de um painel de dados formado pelos 5.565 municípios brasileiros, utilizando o conceito de rede Bayesiana para indicar as relações entre as dimensões da pobreza. Esta abordagem, além de propiciar uma análise baseada na distribuição condicional das variáveis, relaxará as restrições feitas ao sentido de causalidade entre as privações por trabalhos que tratam desta temática.

Além desta seção introdutória, este trabalho apresenta mais cinco seções. A segunda discorrerá sobre as relações de causalidade entre as privações. A terceira apresentará o método de redes Bayesianas. A quarta discutirá os resultados. Por fim, a quinta irá expor as considerações finais.

\section{Relações Entre Privações Socias}

Dentre as principais discussões sobre as diversas facetas da pobreza destaca-se a construção de índices de multidimensionalidade para as privações. Tal medida é capaz de oferecer um forte argumento sobre as condições de vida dos indivíduos, bem como indicar quais as carências mais significativas. Contudo, se o objetivo for à formulação de políticas de erradicação da pobreza, tal indicador oferece uma limitação às ações pelo fato de o mesmo não exprimir o conjunto de inter-relações existes entre os diversos níveis de privações que uma sociedade 
possa enfrentar. $O$ entendimento das relações entre as privações pode fomentar argumentos essenciais para o formulador de política econômica, já que o diagnóstico adequado de tal interação pode propiciar uma elevação da eficiência das ações públicas de combate à pobreza.

\subsection{Efeitos da Privação Monetária}

A privação monetária possui em sua essência um estado crônico capaz de influenciar o desenvolvimento dos agentes econômicos. Indivíduos com baixa renda são candidatos passíveis de transtornos no bem-estar, principalmente, aqueles relacionados às práticas escolares, aos serviços de saúde, condições de moradia etc.

A literatura traz evidências sobre a associação entre o baixo nível de renda e a precariedade nas condições de saúde. Segundo Angell (1993), Hurowitz (1993), Smith (1999), Soares (2006) e Case e Deaton (2005), indivíduos com limitações em renda estão sujeitos à carência em saúde, dado que, como destacado por Feinstein (1993), Adler (1993) e Santos et al (2012), existe uma correlação positiva entre as condições de saúde e o nível de renda. Aquelas pessoas cujo padrão monetário apresenta severas restrições normalmente possuem uma condição nutricional desfavorável, vivem em ambientes com estado precário de higiene sanitária e possuem dificuldades na aquisição de serviços de saúde, tais como exames, consultas ou tratamentos e, portanto, estão mais propicias a apresentarem enfermidades.

A associação entre a pobreza monetária e o desempenho educacional é outro ponto bastante evidenciado na análise social. Dificuldades financeiras impõem restrições sobre o processo de qualificação dos indivíduos. Um baixo nível de renda leva os indivíduos a reduzirem seus investimentos em educação. Segundo Bramley e Karley (2005), a nível macro, geralmente observa-se que os países pobres têm baixos níveis de escolaridade e, a nível micro, os filhos de famílias pobres apresentam uma taxa de frequência escolar pouco expressiva. Ainda, de acordo com Blau (1999), Cameron e Heckman (2000), Levy e Duncan (2001) e Duncan e Chase-Lansdale (2000), há evidências de que aquelas famílias com situação financeira mais favorável possuem uma condição educacional superior às famílias mais carentes e que tal gap educacional seria mais expressivo durante os primeiros anos de vida.

\subsection{Efeitos da Privação em Educação}

Evidências dos efetios da educação sobre as privações em renda, bem como sobre aquelas não monetárias, são explorados, principalmente, pela observação dos ganhos oriundos do aumento da produtividade. Reduzir a carência educacional através da qualificação dos individuos propicia um crescimento da produtividade e, consequentemente, pode culminar no aumento potencial dos ganhos esperados, de forma que as demais privações enfrentadas possam ser minimizadas.

A escolaridade pode ser associada a melhores perspectivas de crescimento econômico. Barro (1991), Mankiw et al (1992) e Romer (1990) sugerem que o 
investimento em capital humano é a condição prévia para os países em desenvolvimento absorverem tecnologia e melhorarem seu nível de produtividade, levando-os a um maior rendimento. De forma singular, a educação fornece uma das principais formas de fuga para as armadilhas da pobreza. Psacharopoulos e Patrinos (2002) relatam que os retornos privados da educação são bastante significativos. De acordo com Tilak (2005) e Wilson (2001), um aumento do nível de escolaridade da família é capaz de reduzir significativamente as chances da mesma ser pobre, dado que o efeito da educação sobre o rendimento mostra-se positivo e estatisticamente significativo. Neste sentido, Schultz (1995) alega que a instrução da mãe provoca uma redução no número de indivíduos da família, elevando o rendimento em termos per capita, proporcionando melhores condições de saúde e um maior nível médio de instrução. Assim, aprovisionar um mínimo de educação àquele indivíduo que apresenta sérias privações monetárias pode representar uma forma orientada de investimento, que, no longo prazo, refletirá no atendimento de suas necessidades.

A privação em educação pode provocar dificuldades salutares por vários motivos. A educação inibe a ignorância dos indivíduos quanto aos hábitos de uma vida saudável. Uma melhoria no nível educacional seria responsável por proporcionar um maior cuidado com o consumo de bens e com as condições de higiene, garantindo uma redução da probabilidade de contaminações. Além disso, de acordo com Grossman (1972), a educação pode afetar diretamente a saúde. Pessoas educadas podem alocar melhor seu tempo com atividades relevantes para a saúde, obterem melhores informações sobre a prevenção de doenças, tratamentos e exames médicos como sugere Glied e Lleras-Muney (2003) e Goldman and Smith (2002). Por fim, de forma indireta, a escolaridade proporciona um retorno superior ao observado pelos indivíduos com instrução inferior, permitindo a aquisição de alimentos mais saudáveis, de uma habitação adequada e de um maior volume de serviços de saúde.

\subsection{Efeitos da Privação em Saúde}

Os efeitos provocados por carências em saúde normalmente são tratados a partir de considerações sobre a produtividade. Pessoas doentes se mostram menos produtivas, já que as condições do organismo, como a capacidade física e mental, encontram-se temporariamente debilitadas. Por exemplo, quando uma criança se encontra doente, normalmente ela perde aulas e tem seu rendimento escolar abalado. No caso de um adulto, quando este se encontra enfermo, observa-se uma limitação no ritmo de suas atividades, que culminaria na redução do nível de renda individual.

Neste sentido, a deterioração da saúde teria um impacto direto na produtividade e na oferta de trabalho, e, portanto, sobre a pobreza monetária. Indivíduos desnutridos, com hábitos como alcoolismo, fumo etc. tendem a apresentar um quadro produtivo inferior àqueles com rotinas saudáveis (Buddelmeyer e Cai, 2009). De acordo com Fuchs (2004), melhores condições de saúde implicam em taxas de sobrevivência mais elevadas, principalmente, para lactentes e crianças, o que eleva o PIB per capita, por aumentar a proporção entre trabalhadores e dependentes. Ainda, quanto menores forem às privações em 
saúde entre adultos, maior será à força de trabalho e sua respectiva produtividade. Mesmo em países desenvolvidos, os efeitos da saúde sobre renda podem ser observados quando os trabalhadores mais velhos, por apresentarem estados não salutares, deixam o quadro da força de trabalho de maneira prematura, reduzindo assim a sua renda atual, bem como sua renda na aposentadoria.

As privações em saúde também são vistas como fontes de limitação para o acúmulo de conhecimento. Para Glewwe e Jacoby (1994) a precariedade na saúde é o principal fator de atraso na vida escolar. Uma iniciação escolar tardia reduz a capacidade da criança de aprender e, assim, minimiza a probabilidade de altos retornos provenientes da educação. Neste sentido, Sanchés e Sbrana (2010) e Shashidhar et al (2009) observam que uma doença crônica ou deficiência reduz a probabilidade de entrada e a frequência na escola básica. Por fim, as carências em saúde podem afetar positivamente a taxa de mortalidade, fazendo com que famílias aumentem seu número de filhos e, assim, reduza o investimento per capita em educação.

\subsection{Efeitos das Privações em Habitação}

A habitação tem o potencial de influenciar significativamente os resultados da educação. Em particular, condições precárias de moradia estão associadas com déficits significativos no desempenho escolar. Indivíduos que não possuem residência são acometidos de um local para estudar, inibindo o seu desenvolvimento educacional. Haurin et al (2002) observa que aqueles estudantes que apresentam um melhor desempenho em testes de matemática e português residem em casa própria, sendo tal diferençal de 10\% e $7 \%$, respectivamente. Braconi (2001) relata que os meninos que vivem em casas de propriedade familiar apresentam 8\% mais chances de concluir o ensino médio. Neste sentido, Green e White (1997) verificam que crianças com uma quantidade de anos de estudo superior são aquelas cujos pais não sofrem com carências habitacionais.

Outro efeito das condições de moradia bastante discutido pela literatura é o melhor estado de saúde para os seus residentes. Uma casa construida a partir de material durável reduz a exposição a enfermidades, epidemias e doenças respiratórias. Saegart (2003) relata que as condições precárias em habitação podem contribuir para a transmissão de doenças infecciosas, lesões, sintomas de asma, envenenamento por chumbo e problemas de saúde mental, especialmente entre as crianças. Vandivere et al (2006) destaca que um perigo comum das privações em moradia é o envenenamento por elementos químicos, cujos efeitos incluem a redução do QI e deficiências no crescimento físico e no desenvolvimento neurológico. Ainda, diante de quadro habitacionais precários, Matte et al (2000) observa certa exposição a infestação de baratas, roedores, ácaros e mofo que são fatores de risco a saúde.

Por fim, pode-se dizer que a privação de residência também pode afetar a situação monetária dos indivíduos. Boehm e Schlottmann (2001) afirmam que a propriedade de uma residência representa a maior fonte de acumulação de riqueza para a maioria das famílias, principalmente entre aquelas com baixa renda. Segundo Knox et al (2000), uma residência possibilita ao individuo um maior bem 
estar, que proporciona ganhos de produtividade e, consequentemente, eleva seus proventos monetários.

Portanto, o que se pode inferir destes argumentos é que a literatura econômica tem destacado um conjunto de inter-relações entre as distintas formas de privações e que as ações que visam minimizar o patamar de uma carência especifica é capaz de afetar, também, as demais dimenssões da pobreza, dado o ciclo de armadilhas que é formado em torno das mesmas. Assim, o dignóstico de tal interação é fudamental para a compreensão dos potenciais que podem ser atigindos a partir das políticas de combate a pobreza.

\section{Metodologia}

$\mathrm{Na}$ tentativa de verificar as relações existentes entre as diversas dimensões da pobreza será utilizada a análise de redes bayesianas. Tal técnica permitirá identificar as inter-relações entre os distintos níveis de pobreza sem admitir de forma ad hoc qualquer sentido causalidade entre as variáveis e, ainda, trabalhará a partir de informações sobre as distribuições condicionais, de modo a garantir um maior nível de profundidade a análise e uma melhor caracterização das relações.

\subsection{Redes Bayesianas}

A Rede Bayesiana é uma estrutura baseada na análise de grafos direcionais que permitem representar as possíveis relações existentes entre um conjunto de variáveis em condições de incerteza. Basicamente, tal ferramenta implementa um algoritmo de aprendizagem num conjunto de variáveis aleatórias, denominadas nós, expressando através de arcos direcionados a dependência direta entre as mesmas a partir das distribuições de probabilidade condicional.

Formalmente, considerando um conjunto de $n$ variáveis aleatórias $X_{1}, X_{2}, \cdots, X_{n}$, um grafo acíclico direcionado com $n$ nós numerados, e, ainda, que o $j$ nó está associado a $X_{j}$. Então, o grafo é uma Rede Bayesiana, que representa as variáveis $X_{1}, X_{2}, \cdots, X_{n}$, se

$$
P\left(X_{1}, X_{2}, \cdots, X_{n}\right)=\prod_{j=1}^{n} P\left(X_{j} / X_{-j}\right)
$$

onde $X_{-j}$ indica o conjunto de todas as variáveis $X_{i}$, de tal modo que há um arco do nó i para o nó j no grafo.

A estrutura da rede captura as relações qualitativas e quantitativas entre as variáveis. Em particular, dois nós são ligados diretamente se um afeta o outro, tendo um arco indicativo para a direção do efeito e o escore de probabilidade como medida para tal efeito. Esquematicamente, uma rede bayesiana pode ser expressa como segue 
Figura 1 - Rede Bayesiana

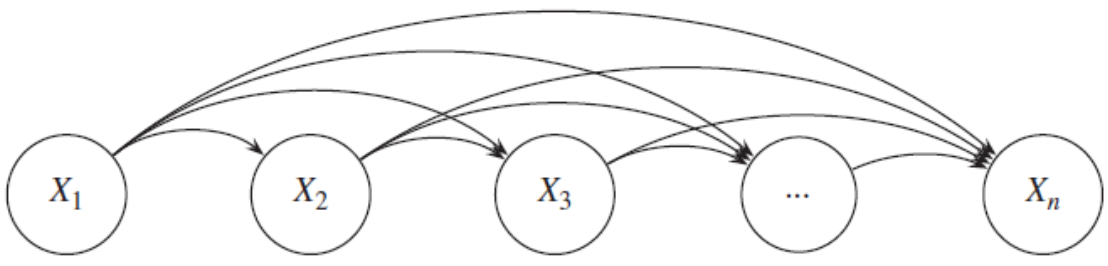

Fonte: Pourret (2008).

Como pode ser observado, a rede Bayesiana apresenta um conjunto de ligações entre as variáveis. Cada nó pode ser conectado direto e/ou indiretamente a outros nós, sendo que aquele nó que não sucede de nenhum outro é denominado de raiz, enquanto que aqueles que possuem nós antecessores ou sucessores são denominados nós intermediários e, por fim, aqueles que não possuem sucessores são os nós finais.

Todas as ligações de uma rede bayesiana são expressas por meio de arcos cujas direções e intensidades são provenientes da relação de dependência entre as distribuições de probabilidades condicionais. No entanto, um elemento fundamental da estrutura da rede é que ela não impõe a existência de ligações entre todos os nós existentes, mas sim, a partir da análise da distribuição de probabilidade conjunta, expressa a mais compacta forma de relação entre as variáveis.

Por fim, a determinação da probabilidade descrita em (1) é obtida pelo algoritmo de construção, o qual simplesmente processa cada nó em ordem, adicionando-o à rede existente e adição de arcos de um conjunto mínimo de nós raiz ou intermediários de tal forma que o conjunto dos nós torna a corrente condicionalmente independente de qualquer outro nó que o precede.

Esquematicamente, o algoritmo de construção para uma rede Bayesiana pode ser idealizado a partir dos seguintes passos:

1. Escolha o conjunto de variáveis relevantes $\left\{X_{i}\right\}$ que descrevem o domínio.

2. Escolha uma ordenação para as variáveis, $\left\langle X_{1}, X_{2}, \ldots, X_{n}\right\rangle$.

3. Enquanto existir variáveis:

(a) Adicione a variável $X_{i}$ a rede.

(b) Adicione arcos ao nó $X_{i}$ de algum conjunto mínimo de nós pertencente a rede, de tal modo que a seguinte propriedade independência condicional seja satisfeita:

$$
P\left(X_{j} / X_{2}{ }^{\prime}, \cdots, X_{n}{ }^{\prime}\right)=P\left(X_{j} / X_{-j}\right)
$$

onde $X_{2}{ }^{\prime}, \cdots, X_{n}{ }^{\prime}$ são todas as variáveis que precedem $X_{j}$.

(c) Defina a probabilidade condicional para $X_{i}$. 
Existem argumentos extremamente favoráveis à construção de uma rede da forma mais parcimoniosa possível. Primeiro, o modelo mais compacto exigirá menos especificações para os valores das probabilidades, promovendo menor esforço computacional. Em segundo lugar, as redes demasiadamente densas não conseguem representar independências explicitamente. Enfim, as redes mais densas falham na busca das dependências causais no domínio.

\subsection{Descrição dos Dados e Expectativas para as Relações}

A amostra utilizada forma um painel com dados sobre quatro óticas da pobreza, quais sejam renda, educação, saúde e habitação, para 5.565 municípios do Brasil no período entre 1970 a 2010. Os dados foram retirados dos censos de 1970, 1980, 1991, 2000 e 2010 do Instituto Brasileiro de Geografia e Estatística IBGE, formando um painel com 5565 municípios em 5 anos, com intervalo temporal decenal característico dos censos. É fato que alguns municípios não existiam, especialmente durante os 3 primeiros censos. Contudo, seus respectivos valores foram tomados como ausentes na formação do painel e, apesar disto, o painel se mostrou balanceado. A tabela 1 traz mais informações sobre a natureza dos dados utilizados.

Tabela 1 - Relação das variáveis utilizadas nas estimações.

\begin{tabular}{l|l}
\hline \multicolumn{1}{c|}{ Variável } & \multicolumn{1}{c}{ Descrição } \\
\hline Pobreza em Renda & Percentual de pessoas pobres (Po). \\
Pobreza em Educação & Percentual de analfabetos 15 ou mais anos. \\
Pobreza em Saúde & Mortalidade infantil por mil vivos. \\
Pobreza em Habitação & Percentual de domicílios construídos sem material durável. \\
\hline \multicolumn{2}{l}{ Fonte: elaboração própria. }
\end{tabular}

Espera-se que a pobreza monetária influencie as demais condições sócias, já que a mesma é capaz de inibir a capacidade de o indivíduo adquirir qualificações, serviços de saúde e uma unidade habitacional. Ainda, admite-se que possa existir uma relação direta entre as carências em educação e renda, pelo fato de uma maior produtividade representar acréscimos ao fluxo de rendimentos de um indivíduo. Por fim, é razoável supor que uma melhor condição de moradia permita um melhor aparato de prevenção de doenças.

\section{Resultados}

Para compreender o comportamento interativo entres privações sociais e, assim, entender suas respectivas influências sobre as capacidades e limitações que os agentes enfrentam para superar suas carências, optou-se por uma análise baseada nas distribuições condicionais. Tal abordagem, além de permitir uma apreciação mais minuciosa, também propicia um exame categoricamente mais adequado, já que a mesma incorpora condições formadas a partir das demais variáveis estudadas às análises de causalidade individuais.

Para compreender melhor o cenário das privações sociais enfrentadas pelos municípios brasileiros, primeiramente serão apresentadas algumas 
estatísticas descritivas; em seguida, apresenta-se a rede bayesiana construída a partir das quatro óticas da pobreza estudadas.

\subsection{Análise Descritiva das Privações}

Para identificar padrões iniciais sobre o comportamento das privações municipais foi construída uma série de estatísticas descritivas. Tais medidas são responsáveis por apresentar um diagnóstico inicial para o conjunto de informações trabalhadas neste estudo, o qual servirá de base para os argumentos da análise dependência. A tabela 2 apresenta o referido conjunto de estatísticas, para cada ano censitário e para o painel de dados formado pelos últimos 5 censos.

Tabela 2 - Estatísticas descritivas para as dimensões da pobreza.

\begin{tabular}{|c|c|c|c|c|c|c|c|c|}
\hline Período & Privação & $\begin{array}{c}\text { Médi } \\
\text { a }\end{array}$ & $\begin{array}{c}\text { Desvio } \\
\text { Padrã } \\
\text { o }\end{array}$ & $\begin{array}{c}\text { Coeficient } \\
\text { e de } \\
\text { Variação }\end{array}$ & $\begin{array}{c}\text { Assimetri } \\
\mathrm{a}\end{array}$ & $\begin{array}{c}\text { Curtos } \\
\text { e }\end{array}$ & $\begin{array}{c}\text { Máxim } \\
\text { o }\end{array}$ & $\begin{array}{c}\text { Mínim } \\
\text { o }\end{array}$ \\
\hline \multirow{4}{*}{$\begin{array}{l}1970 / 201 \\
0\end{array}$} & Renda & 49,93 & 29,55 & 0,59 & $-0,05$ & 1,70 & 100 & 0,34 \\
\hline & Saúde & 62,69 & 44,90 & 0,71 & 1,80 & 6,78 & 30,66 & 3,09 \\
\hline & $\begin{array}{l}\text { Habitaçã } \\
\text { o }\end{array}$ & 65,38 & 30,02 & 0,46 & $-0,61$ & 2,06 & 100 & 0,00 \\
\hline & Educação & 38,33 & 20,19 & 0,53 & 0,14 & 1,81 & 92,40 & 0,91 \\
\hline \multirow{4}{*}{1970} & Renda & 83,96 & 14,88 & 0,18 & $-1,79$ & 6,96 & 100 & 7,68 \\
\hline & Saúde & 12,36 & 5,28 & 0,43 & 0,94 & 3,26 & 30,37 & 2,79 \\
\hline & $\begin{array}{l}\text { Habitaçã } \\
\text { o }\end{array}$ & 68,34 & 22,94 & 0,34 & $-0,81$ & 2,91 & 100 & 0,80 \\
\hline & Educação & 43,65 & 18,25 & 0,42 & 0,18 & 1,99 & 92,40 & 1,10 \\
\hline \multirow{4}{*}{1980} & Renda & 56,25 & 22,71 & 0,40 & $-0,21$ & 2,08 & 97,91 & 1,69 \\
\hline & Saúde & 85,31 & 44,85 & 0,53 & 1,25 & 3,79 & 100 & 21,7 \\
\hline & $\begin{array}{l}\text { Habitaçã } \\
\text { o }\end{array}$ & 79,47 & 23,43 & 0,29 & $-1,33$ & 4,01 & 100 & 0,30 \\
\hline & Educação & 36,74 & 18,06 & 0,49 & 0,35 & 1,96 & 91,40 & 2,90 \\
\hline \multirow{4}{*}{1991} & Renda & 58,50 & 23,06 & 0,39 & $-0,39$ & 2,04 & 95,89 & 1,62 \\
\hline & Saúde & 49,45 & 24,99 & 0,51 & 0,71 & 2,59 & 100 & 10,65 \\
\hline & $\begin{array}{l}\text { Habitaçã } \\
\text { o }\end{array}$ & 87,49 & 17,98 & 0,21 & $-2,08$ & 7,08 & 100 & 8,50 \\
\hline & Educação & 31,16 & 16,91 & 0,54 & 0,48 & 2,08 & 89,20 & 1,80 \\
\hline \multirow{4}{*}{2000} & Renda & 46,47 & 22,85 & 0,49 & 0,02 & 1,64 & 93,02 & 2,74 \\
\hline & Saúde & 34,08 & 18,47 & 0,54 & 0,72 & 2,68 & 100 & 5,38 \\
\hline & $\begin{array}{l}\text { Habitaçã } \\
\text { o }\end{array}$ & 86,94 & 16,46 & 0,19 & $-1,64$ & 5,22 & 100 & 8,07 \\
\hline & Educação & 21,77 & 12,46 & 0,57 & 0,58 & 2,24 & 60,70 & 0,90 \\
\hline \multirow{4}{*}{2010} & Renda & 40,89 & 12,00 & 0,29 & 0,14 & 2,22 & 75,98 & 9,56 \\
\hline & Saúde & 22,08 & 13,06 & 0,59 & 1,10 & 15,69 & 100 & 0,00 \\
\hline & $\begin{array}{l}\text { Habitaçã } \\
\text { o }\end{array}$ & 82,46 & 22,37 & 0,27 & $-1,39$ & 4,07 & 100 & 0,38 \\
\hline & Educação & 16,35 & 4,01 & 0,25 & 0,41 & 2,27 & 30,40 & 7,65 \\
\hline
\end{tabular}

Fonte: elaboração própria.

De acordo com a tabela dois, pode-se verificar um valor 49,93\% para a percentual de pessoas pobres. Tal fato indica que entre 1970 e 2010, em média, quase metade da população residente nos municípios brasileiros apresentava 
pobreza monetária. Pontualmente, observando cada censo, verifica-se que a situação da privação monetária vem sendo suavizada, reduzindo-se de $84 \%$, em 1970, da população para 40,89\%, em 2010. Ainda, destaca-se que a referida medida apresenta uma dispersão de 29,55 unidades, para média do período, mas com um patamar mais homogêneo em 2010, cuja dispersão encontra-se em apenas 12 unidades. Apesar da gravidade apresentada por tais estatísticas, as privações monetárias não se mostram significativas em alguns municípios, já que a amostra contempla cidades com Po inferior a 5\%, sendo seu valor mínimo de 0,34\%. Por fim, verifica-se uma distribuição municipal praticamente simétrica e uma curtose do tipo leptocúrtica, o que define a distribuição da pobreza monetária de forma concentrada em torno da média, mas com um leve afastamento à direita e com frequências relativamente baixas, o que reforça a forte dispersão existente na série.

Analisando a ótica da pobreza em educação, verifica-se um percentual de analfabetos, em média, igual 38,33\%, com uma dispersão em torno da mesma de $53 \%$, considerando todos os censos. Assim como no caso monetário, o resultado para educação também decresce substancialmente ao longo das décadas, atingindo no último censo a patamar de $16,35 \%$ da população. Assim, embora, no intervalo 1970-2010, pouco mais de um terço da população residente nas cidades do Brasil com idade superior a 15 anos possuírem privações educacionais, podendo este valor alcançar o patamar de 92,4\% do total de residente, o último censo deixa claro que este tipo de privação tem sido amenizado e o máximo de indivíduos analfabetos em um município situa-se em torno de 30\% como mostra à estatística máximo. As estatísticas de assimetria e curtose indicam que a distribuição municipal de privações em educação apresenta-se com um afastamento a esquerda e na forma leptocúrtica, o que reflete um comportamento muito próximo ao normal, ou seja, com uma média muito próxima ao centro e com frequências distribuídas de forma homogênea entre as classes. Assim, o que se pode observar para realidade municipal no Brasil é que as privações em educação são muito semelhantes, já que a distribuição observada para a série é muito próxima da variante Normal.

Com uma média de 62,97\% e um dispersão de 44,9, sendo esta última cerca de $71 \%$ em termos da média, tem-se que a situação municipal em termos de condições de saúde apresenta um estado bastante precária. Estes resultados indicam que, a cada cem mil nascidos vivos, têm-se 68 óbitos, em alguns casos chegando a 303. Ao longo dos censos, a referida estatística apresenta um cenário mais otimista, chegando a atingir em 2010 1/4 da mortalidade observada em 1970. Contudo, em termos absolutos, são observados municípios com até 100 óbitos por 1000 mil nascidos vivos. A referida série apresenta um grau de afastamento a esquerda e uma curtose do tipo leptocúrtica, refletido que a média amostral é inferior ao valor modal e que as frequências para as observações se mostram bastantes elevadas, ou seja, acima dos valores normais. Por um lado, o fato de a distribuição apresentar assimetria a esquerda indica que a média de mortalidade infantil é inferior ao número de casos mais frequentes na amostra. Por outro, o fato de um elevado coeficiente de curtose reforça a observação de altas ocorrências para os diversos elementos da amostra. 
No que se refere às privações em habitação, medida pelo percentual de casas construídas com material durável, observa-se que $65,38 \%$ da população residem em domicílios construídos de forma apropriada, ou seja, em condições adequadas de moradia. Com um desvio padrão de 30,02, correspondendo a uma dispersão em torno da média de $46 \%$, pode-se observar uma elevada discrepância nas privações municipais de habitação. Ainda, de acordo com as estatísticas máximo e mínimo, identificou-se cidades que possuem 100\% das suas residências construídas com material durável e, também, casos nos quais este percentual é nulo, o que reforça a acentuada variabilidade neste tipo de privação. Por fim, diferentemente das outras óticas analisadas, não se observa mudanças significativas nos valores médios, especialmente durante os três últimos censos.

Desta forma, as estatísticas descritivas sinalizam uma melhoria dos níveis de privações sociais e, consequentemente, um movimento similar entre as mesmas. Tal condição sugere que as privações possam interagir de forma a produzir um cenário mais favorável a população dos municípios brasileiros.

\subsection{Rede Bayesiana de Privações Sociais}

Como discutido anteriormente, as redes Bayesianas baseiam as relações de causalidades a partir da determinação das probabilidades condicionais. Assim, o que se pode inferir da rede estimada são as relações de causalidade existentes entre as privações dadas suas respectivas distribuições marginais condicionais. Assim, a relação direta entre quaisquer duas medidas de pobreza, na verdade seriam uma medida de causalidade entre as mesmas condicionada aos valores dos demais indicadores de privação. Por exemplo, caso haja uma relação entre o analfabetismo e a proporção de pobres, tal relação indicaria que flutuações no número de analfabetos afetariam a proporção de pobres, ou o inverso, ter-se-ia como fator condicionante as distribuições marginais das carências em saúde e em habitação. A rede Bayesiana que define as relações de causalidade entre as privações monetárias, educacionais, saúde e habitação são apresentadas a seguir. 
Figura 2 - Rede Bayesiana de Privações Sociais
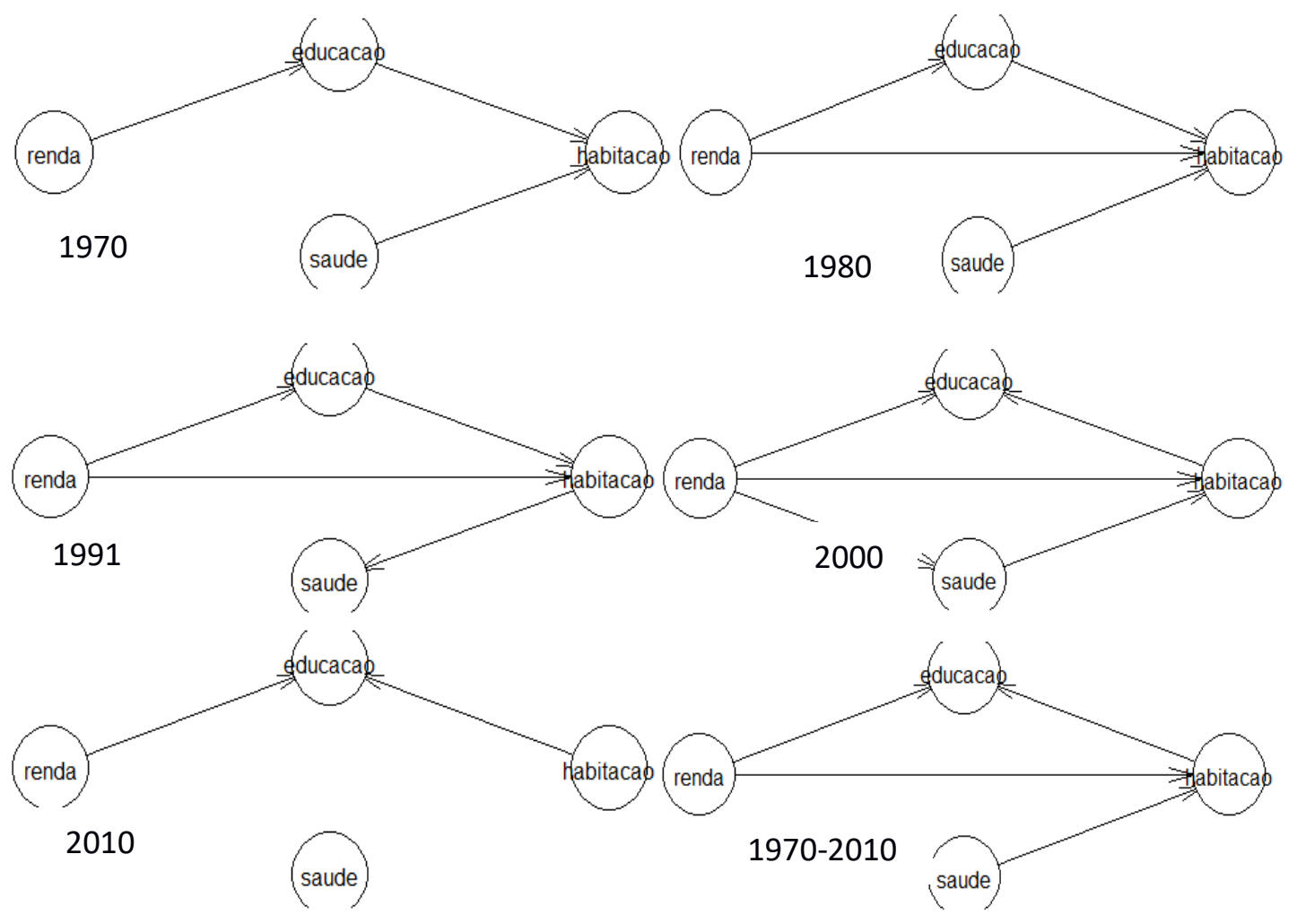

Fonte: elaboração própria.

Dentre as relações observadas para as séries de carências tratadas, o único efeito negativo remete-se a relação entre a condição monetária e a habitacional. Como destaca a figura 2, o sentido para relação se expressa na direção da renda para a habitação e, assim, para cada novo pobre ter-se-ia um impacto negativo no número de casas construídas com material durável. O sentido negativo para relação da condição de moradia e a privação em renda reflete a cenário da política habitacional que garante as pessoas com menores níveis de renda o direito à moradia, seja pela concessão pública ou pelos incentivos ao financiamento imobiliário.

Outro efeito das condições de moradia diz respeito aos seus efeitos sobre o estado de saúde, como observado pela rede estimada para 1991. Nesta direção, verifica-se que melhores condições habitacionais reproduzem um cenário salutar. Indivíduos que possuem sua unidade residencial construída a partir de material durável apresentam uma melhor estrutura sanitária e de higiene, o que reflete diretamente no combate a proliferação de infecções, doenças respiratórias e epidemias como destacado por Saegart (2003) e Vandivere et al (2006). Ademais, o sentido inverso prevalece em quase todos os outros períodos (sendo 2000 a exceção) e na amostra formada pelas frequências de todos os períodos. Como destacado por Buddelmeyer e Cai (2009), tal relação pode ser justificada no fato 
das carências em saúde diminuírem a produtividade do indivíduo e, consequentemente, sua capacidade de aquisição da casa própria.

Por fim, a interação entre as carências educacionais e em habitação se mostram de forma alternada. Entre 1970 e 1991, observa-se uma relação onde o nível educacional é capaz de afetar a condição de moradia. Contudo, para os dois últimos censos e, também, ao se considerar o painel de dados formado pelos cinco censos, o sentido para tal relação se inverte. Por um lado, carências em educação privaria o acesso das pessoas a moradias adequadas por limitar o rendimento e, assim, a capacidade de aquisição da casa própria. Por outro, como destacado por Braconi (2001) e Green e White (1997), uma melhor condição de moradia propicia um ambiente mais adequado ao aprendizado e, consequentemente, elevam a probabilidade do indivíduo concluir o ensino médio e/ou superior.

A carência em saúde também apresenta relações com as privações monetárias. Locais com elevado número de pobres, ou seja, com carências em renda exacerbadas, tendem a apresentar um conjunto de problemas imunológicos. Assim como exposto por Feinstein (1993), Adler (1993), Soares (2006) e Santos et al (2012), indivíduos com sérias restrições monetárias sofrem com estados nutricionais desfavoráveis, convivem com precariedades de higiene e dificilmente têm acesso a serviços de saúde, encontrando-se, portanto, passíveis de enfermidades.

Ainda, a relação observada para as carências monetária e em saúde indica que as variações nos rendimentos estão integradas as melhorias nas condições de saúde, ainda que está relação não se mostre de forma direta como observado em 2000. Quanto menores forem às privações em renda, melhores serão as condições educacionais e de moradia e, consequentemente, o estado de saúde dos indivíduos. Um menor contingente de pessoas pobres revela uma situação na qual indivíduos são mais qualificado e residem numa propriedade mais adequada, reduzindo a ignorância a tratamentos, possibilitando uma alimentação mais saudável e melhores condições de higiene, refletindo um estado mais salutar. Ainda, especialmente para o ano de 2000, é possível observar um efeito direto da carência monetária sobre a condição de saúde. Quanto menor o número de pobres, maiores serão as oportunidades de aquisição de serviços de saúde, possibilitando uma redução deste tipo de carência. Um nível de renda muito baixo restringe a aquisição de serviços clínicos como consultas, tratamentos, cirurgias, medicamentos etc., bem como de alimentos e práticas mais salutares, inibindo a adoção de um padrão de vida mais saudável. Resultados semelhantes são vistos em Soares (2006).

Finalmente, a relação entre as carências em educação e saúde podem ser expressas nos dois sentidos como mostram as redes de 1991 e 2000/1970-2010, respectivamente. Por um lado, o nível de escolaridade dos indivíduos permite que os mesmos se tornem menos ignorantes quanto a uso de tratamentos médicos e, principalmente, quanto à prevenção de doenças. Segundo Grossman (1972), pessoas mais educadas alocam melhor seu tempo com atividades relevantes para a saúde, obtêm melhores informações sobre a prevenção de doenças, tratamentos e exames médicos, como também sugerem Glied e Lleras-Muney (2003) e Goldman and Smith (2002). Por outro, como observado por Sanchés e Sbrana (2010) e Shashidhar et al (2009), um estado mais salutar permite a 
adequação idade-série, maior frequência escolar e uma condição fisiológica mais favoravel ao aprendizado. Portanto, uma situação de saúde mais favorável seria condizente com um melhor nível de educação, como observado para o ano 2000 e para a amostra como um todo.

Avaliadas as relações existentes entre as dimensões de pobreza, é possível inferir sobre o rumo a ser adotado nas políticas de combate as carências sociais, de modo que as mesmas alcancem resultados mais eficientes e, assim, melhorem as condições do brasileiro. Políticas voltadas para o desenvolvimento educacional, transferências de renda e a questão habitacional são capazes de influenciar o estado da saúde. Assim, quando se busca elevar o nível de renda e propiciar a aquisição de unidades residenciais aos indivíduos, além de reduzir a carência monetária e melhorar a condição de moradia, pode-se observar melhorias na saúde e, principalmente, quando se investe em educação se observa um melhoramento do quadro geral da pobreza.

Do ponto de vista individual, os resultados da rede Bayesiana permitem inferir que as condições de pobreza determinam o estado geral de carência. Indivíduos analfabetos tendem a serem pobres em saúde e em moradia. Ainda, aqueles com privações monetárias têm maiores prejuízos à saúde, moradias inadequadas e um nível de educação inferior. Por fim, a condição de moradia também é capaz de influenciar o padrão educacional e de saúde do indivíduo. Portanto, aquele indivíduo que apresenta algum tipo de carência provavelmente arcará com outras formas de privações. Ou seja, não há nada tão ruim que não possa piorar.

\section{Considerações finais}

Explorar as relações entre distintos níveis de privação, embora pouco explorado pela literatura, exige categoricamente uma proposta que não restrinja o sentido de causalidade para as relações e, ainda, contemple um detalhamento adequado para a distribuição. Diante de tais requisitos, a abordagem de redes Bayesianas se mostra uma ferramenta de análise adequada.

A estimação da rede de privações baseada na proposta Bayesiana de analisar as relações através da distribuição condicional permite qualificar as condições dos indivíduos e, assim, entender o processo pelo qual um dado agente encontra-se por apresentar um dado conjunto de limitações. Deste modo, os resultados da rede Bayesiana possibilitaram a compreensão das interações entre as privações municipais e ajudaram nos argumentos em prol do entendimento do atual estado de privação.

Como esperado, há uma série de relações entre os diferentes tipos de pobreza e, portanto, a compreensão do comportamento de uma dada medida de privação necessariamente passa pelo entendimento das demais formas de carências enfrentadas por uma sociedade. Definitivamente, entender a privação monetária requer uma análise sobre a produtividade; compreender a situação da saúde sobrevém das informações sobre a limitação monetária, do nível de escolaridade e da condição de moradia; enfim, o padrão de uma unidade residencial é afetado diretamente pela condição financeira dos agentes, assim como suas condições de saúde e de educação. 
Tal fato reflete a importância que o diagnóstico das relações entre carências exerce sobre a tomada de decisão pública no combate à pobreza. É preciso entender como se propagam as ações públicas de forma que o resultado observado para dada política permita melhorias de bem-estar. Portanto, sabendo que os principais efeitos se propagam da educação e da renda para a saúde e para habitação, elevar a renda dos indivíduos e, principalmente, seu nível de escolaridade, permite, além de melhorias na própria situação monetária e educacional, a redução das precariedades em saúde e do número de famílias sem uma residência digna.

Por fim, apesar de não ser observado nenhum indício sobre os efeitos que as condições de saúde exercem sobre as demais formas de privação, tal resultado não permite inferir que eventuais ações em prol do melhoramento do sistema de saúde não são necessárias, mas sim que elas são as menos indicadas se o objetivo for à melhoria nas condições diversas de pobreza.

\section{REFERÊNCIAS}

ADLER, Nancy E. Socioeconomic inequalities in health: no easy solution. Jama, v. 269, n. 24, p. 3140-3145, 1993.

ALKIRE, Sabina; FOSTER, James. Counting and multidimensional poverty measurement. Journal of public economics, v. 95, n. 7, p. 476-487, 2011.

ANGELL, Marcia. Privilege and health-what is the connection?. The New England journal of medicine, v. 329, n. 2, p. 126, 1993.

ATKINSON, A.; BOURGUIGNON, F.: The comparison of multidimensioned distributions of economic status. Review Economics Studies. 49, p.183-201, 1982.

BARRO, Robert J. Economic growth in a cross section of countries. National Bureau of Economic Research, 1991.

$\mathrm{BIBI}$, Sami. Measuring poverty in a multidimensional perspective: A review of literature. 2005.

BLAU, David M. The effect of income on child development. Review of Economics and Statistics, v. 81, n. 2, p. 261-276, 1999.

BOEHM, T.; SCHLOTTMANN, A. M. Housing and wealth accumulation: Intergenerational impacts (Low-Income Homeownership Working Paper Series, LIHO-01.15). Cambridge, MA: Joint Center for Housing, Harvard University, 2001.

BOSSERT, Walter; CHAKRAVARTY, Satya R.; D'AMBROSIO, Conchita.

Multidimensional poverty and material deprivation. 2009. 
BOURGUIGNON, Francois; CHAKRAVARTY, Satya R. The measurement of multidimensional poverty. The Journal of Economic Inequality, v. 1, n. 1, p. 25-49, 2003.

BRACONI, Frank. Housing and Schooling. The Urban Prospect, v. 7, p. 1-4, 2001.

BRAMLEY, G.; KARLEY, N. Home Ownership, Poverty and Educational Achievement: Individual School and Neighbourhood Effects, paper to the Scottish Executive Education Department. Edinburgh: Centre for Research into Socially Inclusive Services, Heriot Watt University, 2005.

BUDDELMEYER, Hielke; CAI, Lixin. Interrelated dynamics of health and poverty in Australia. IZA Discussion Papers, 2009.

CAMERON, Stephen V.; HECKMAN, James J. The dynamics of educational attainment for black, hispanic, and white males. Journal of political Economy, $v$. 109, n. 3, p. 455-499, 2000.

CASE, Anne; DEATON, Angus. Health and wealth among the poor: India and South Africa compared. American Economic Review, p. 229-233, 2005.

CERIOLI, Andrea; ZANI, Sergio. A fuzzy approach to the measurement of poverty. In: Income and wealth distribution, inequality and poverty. Springer Berlin Heidelberg, 1990. p. 272-284.

CHAKRAVARTY, Satya; DEUTSCH, Joseph; SILBER, Jacques. On the Watts Multidimensional Poverty Index and its Decomposition. World Development 36(6): 1067-1077. 2008.

CHELI, Bruno; LEMMI, Achille. A" Totally" fuzzy and relative approach to the multidimensional analysis of poverty. ECONOMIC NOTES-SIENA-, p. 115-134, 1995

DUCLOS, J.; SAHN, D.; YOUNGER, E. Robust multidimensional poverty comparisons. Economic Journal, 116 (514): 943-968. 2006.

DUNCAN, G.; CHASE-LANSDALE, L. Wefare Reform and Child Well-being. Mimeo Northwestern University, 2000.

FEINSTEIN, Jonathan S. The relationship between socioeconomic status and health: a review of the literature. The Milbank Quarterly, p. 279-322, 1993.

FUCHS, Victor R. Reflections on the socio-economic correlates of health.Journal of health economics, v. 23, n. 4, p. 653-661, 2004.

GLEWWE, Paul; JACOBY, Hanan. Student achievement and schooling choice in lowincome countries: Evidence from Ghana. Journal of Human Resources, p. 843-864, 1994. 
GLIED, Sherry; LLERAS-MUNEY, Adriana. Health inequality, education and medical innovation. National Bureau of Economic Research, 2003.

GOLDMAN, Dana P.; SMITH, James P. Can patient self-management help explain the SES health gradient?. Proceedings of the National Academy of Sciences, v. 99, n. 16, p. 10929-10934, 2002.

GREEN, Richard K.; WHITE, Michelle J. Measuring the benefits of homeowning: Effects on children. Journal of Urban Economics, v. 41, n. 3, p. 441-461, 1997.

GROSSMAN, Michael. The demand for health: a theoretical and empirical investigation. NBER Books, 1972.

HAURIN, Donald R.; PARCEL, Toby L.; HAURIN, R. Jean. Does homeownership affect child outcomes?. Real Estate Economics, v. 30, n. 4, p. 635-666, 2002.

HUROWITZ, James C. Toward a social policy for health. The New England journal of medicine, v. 329, n. 2, p. 130-133, 1993.

KNOX, Virginia W.; MILLER, Cynthia; GENNETIAN, Lisa A. Reforming welfare and rewarding work: A summary of the final report on the Minnesota Family Investment Program. New York: Manpower Demonstration Research Corporation, 2000.

KOLM, S-Ch. Multidimensional egalitarianisms. Quartely Journal Economics. 91: 113, 1977.

LEVY, D.; DUNCAN, G. Using siblings to assess the effect of childhood family income on completed schooling. Joint Centre for Poverty Research Working Paper, North Western University, 2001.

MATTE, Thomas D.; JACOBS, David E. Housing and health—current issues and implications for research and programs. Journal of Urban Health, v. 77, n. 1, p. 7-25, 2000.

MAASOUMI, E. The Measurement and Decomposition of Multidimensional Inequality, Econometrica, 54, 771-779, 1986.

MAASOUMI, Esfandiar; LUGO, Maria Ana. The Information Basis of Multivariate Poverty Assessments. In Quantitative Approaches to Multidimensional Poverty Measurement, Kakwani and Silber (eds.), Palgrave Macmillan, 2008.

MANKIW, N. Gregory; ROMER, David; WEIL, David N. A contribution to the empirics of economic growth. National Bureau of Economic Research, 1992. 
PSACHAROPOULOS, George.; PATRINOS, Harry A. Returns to investment in education: a further update. World Bank Policy Research Working Paper Series, Vol, 2002.

ROMER, Paul M. Endogenous technological change. Journal of political Economy, p. S71-S102, 1990.

RUGGIERI-LADERCHI, C.; SAITH, R.; STEWART, F. Does it matter that we do not agree on the definition of poverty? A comparison of four approaches. Oxford Development Studies, 31, 243-274. 2003.

SAEGERT, Susan C. et al. Healthy housing: a structured review of published evaluations of US interventions to improve health by modifying housing in the United States, 1990-2001. American Journal of Public Health, v. 93, n. 9, p. 1471$1477,2003$.

SÁNCHEZ, Marco V.; SBRANA, Giacomo. Determinants of education attainment and development goals in Yemen. Prepared for the Project Assessing Development Strategies to achieve the Millennium Development Goals in the Arab Region, UNDP-RBAS, UN-DESA and World Bank [downloadable from: http://www.un.org/en/development/desa/policy/capacity/yemen.shtml], 2009.

SANTOS, Anderson Moreira Aristides dos; JACINTO, Paulo de Andrade; TEJADA, Cesar Augusto Oviedo. Causalidade entre renda e saúde: uma análise através da abordagem de dados em painel com os estados do Brasil. Estudos Econômicos (São Paulo), v. 42, n. 2, p. 229-261, 2012.

SCHULTZ, T. Paul. Accounting for public expenditures on education: an international panel study. 1995.

SEN, Amartya K. Poverty: An ordinal approach to measurement. Econometrica. 44 (2): 219-231. 1976.

SHASHIDHAR, Saraswati; RAO, Chandrika; HEGDE, Radhakrishna. Factors affecting scholastic performances of adolescents. The Indian Journal of Pediatrics, v. 76, n. 5, p. 495-499, 2009.

SMITH, James P. Healthy bodies and thick wallets: the dual relation between health and economic status. The journal of economic perspectives: a journal of the American Economic Association, v. 13, n. 2, p. 144, 1999.

SOARES, R. R. The effect of longevity on schooling and fertility: evidence from the Brazilian Demographic and Health Survey. Journal of Population Economics, v.19, n.1, p. 71-97, n.1, 2006.

TILAK, Jandhyala BG. Post-elementary education, poverty and development in India y Role of post-basic education in alleviation of poverty and 
development.Centre of African Studies, University of Edinburgh. Disponível em : www.cas.ed.ac.uk/research/projects. html\# pubs [02/05/2007], 2005.

TSUI, Kai-yuen. Multidimensional Generalizations of the Relative and Absolute Indices: the Atkinson-Kolm-Sen Approach. Journal of Economic Theory, (1995), 67, 251-265, 1995.

TSUI, Kai-yuen. Multidimensional poverty indices. Social Choice and Welfare, v. 19, n. 1, p. 69-93, 2002.

VANDIVERE, S.; HAIR, E. C.; THEOKAS, C.; CLEVELAND, K.; MCNAMARA, M.; ATIENZA, A. How housing affects child well-being. Funders' Network for Smart Growth and Livable Communities, 2006.

WILSON, Kathryn. The determinants of educational attainment: Modeling and estimating the human capital model and education production functions.

Southern Economic Journal, p. 518-551, 2001.

Rodolfo Ferreira Ribeiro da Costa Doutor em Economia pelo CAEN/UFC. Universidade do Estado do Rio Grande do Norte. Professor Adjunto IV. Av. Francisco Mota, 4222, Lote B1-16, Rincão. Mossoró/RN. CEP: 59.626-105. E-mail: rodolfocosta@uern.br/rodolfofrc@yahoo.com.br

Como citar: COSTA, Rodolfo Ferreira Ribeiro da. Não há nada tão ruim que não possa piorar: uma análise bayesiana da pobreza. Redes (St. Cruz Sul, Online), Santa Cruz do Sul, v. $25, \quad$ p. 1933-1952, out. 2020. ISSN 1982-6745. doi:https://doi.org/10.17058/redes.v25i4.12685.

Fontes de financiamento: CAPES e CNPq. 